\title{
COMMENT
}

\section{The smallest nanowire spectrometers}

\author{
Jianji DONG (ه) \\ Wuhan National Laboratory for Optoelectronics, School of Optical and Electronic Information, \\ Huazhong University of Science and Technology, Wuhan 430074, China
}

(C) Higher Education Press and Springer-Verlag GmbH Germany, part of Springer Nature 2019

Optical spectroscopy is a versatile characterization technique for a wide range of applications. Developing miniaturized spectrometers is the trend for applications in which small footprint takes precedence over high resolution. However, development of micro-spectrometers based on miniaturized or integrated optics is approaching a bottleneck toward submillimeter scales because of the inherent scale limitation of their optical components or path lengths. Although these constraints can be circumvented with computational spectral reconstruction by addressing a full range of spectral components simultaneously at multiple detectors, complex millimeter-scale arrays of individually prepared filters arranged over charge-coupled device or complementary metal-oxide semiconductor detectors are difficult to be miniaturized.

By compositionally engineering a single nanowire and electronically probing the photocurrent at a series of points along the nanowire, Zongyin Yang and his co-workers from Cambridge University [1] designed and demonstrated an ultracompact microspectrometer, which is capable of accurate computational spectrum reconstruction in the visible-range. The entire active element of the spectrometer is scaled down to a footprint of just hundreds of nanometers in width and tens of micrometers in length. By means of a spatial point-scanning strategy, the nanowire spectrometer can realize spectral imaging for applications in many fields, such as astronomy and bio-detection. Furthermore, they demonstrate lensless, single-cell-scale in situ spectral imaging by a shift register strategy, which is benefited by the microscale nanowire spectrometer units. This work could open new opportunities for many miniaturized spectroscopic applications.

\section{Reference}

1. Yang Z, Albrow-Owen T, Cui H, Alexander-Webber J, Gu F, Wang X, Wu T C, Zhuge M, Williams C, Wang P, Zayats A V, Cai W, Dai L, Hofmann S, Overend M, Tong L, Yang Q, Sun Z, Hasan T. Singlenanowire spectrometers. Science, 2019, 365(6457): 1017-1020
Received November 28, 2019

E-mail: jjdong@mail.hust.edu.cn

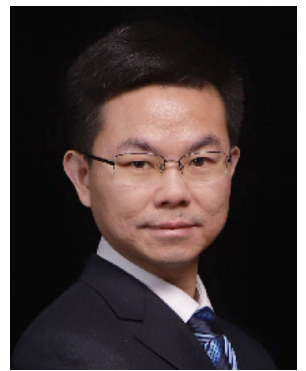

Jianji Dong is Professor of Wuhan National Laboratory for Optoelectronics (WNLO), Huazhong University of Science and Technology (HUST). He received his Ph.D. degree of Optical Engineering at HUST in 2008. After that, he worked as postdoc at Cambridge University, UK till 2010. From March 2010, he returned to HUST and was promoted as a full professor in 2013. His research interests include microwave photonics, silicon photonics, and photonic computing. He has published more than 100 Journal papers, including Nature Communications, Light: Science and Applications, Physical Review Letters, Optics Express, etc. He has some special contribution to energyefficient graphene silicon microheater and complex spectrum analyzer of orbital angular momentum mode. He was honored the Fund of Excellent Youth Scholar by the National Natural Science Foundation of China (NSFC), and honored First award of Natural Science of Hubei Province. He is the editorial member of Scientific Reports, associate editor of IET Optoelectronics and executive editor-in-chief of Frontiers of Optoelectronics. 